\title{
Edge states in Schwinger-boson mean-field theory of low-dimensional quantum antiferromagnets
}

\author{
T. K. Ng \\ Department of Physics, Hong Kong University of Science and Technology, Clear Water Bay Road, Hong Kong
}

(Received 15 January 1993)

\begin{abstract}
The origin and properties of the $S=\frac{1}{2}$ end states in $S=1$ open spin chains is explained within the framework of Schwinger-boson mean-field theory. Moreover, we show that in two dimensions (square lattice), corresponding edge states exist with structures depending on spin magnitude $S$.
\end{abstract}

Recently there has been much interest in the study of finite one-dimensional quantum spin chains following the discovery of fractional $S=\frac{1}{2}$ spin states localized at the ends of $S=1$ spin chains. ${ }^{1,2}$ In a recent paper, ${ }^{3}$ we showed numerically that the $S=\frac{1}{2}$ spin states can be understood within the framework of the Schwinger-boson mean-field theory (SBMFT) for the Heisenberg model. ${ }^{4,5}$ In this paper, we shall derive an analytic explanation within the SBMFT framework for the end states. We shall show that corresponding to the generation of end states, a weak (spin) dimerization effect occurs near the ends of spin chains. The situation for general (integer) values of spin $S$ will be discussed. Furthermore, we shall show using a similar analysis in two dimensions (2D) that corresponding edge states also exist in $2 \mathrm{D}$ quantum antiferromagnets (on a square lattice) with structures depending periodically on spin value $S$.

It is helpful to first review some basic physics of the edge states in $S=1$ quantum spin chains. It is generally believed that the qualitative behavior of the $S=1$ spin chain can be understood in terms of the valence-bondsolid (VBS) $\operatorname{model}^{6}$ where $S=1$ spin on each site is represented by symmetrization of two $S=\frac{1}{2}$ spins and a many-body singlet ground-state wave function is formed by making two valence bonds ${ }^{6-8}$ from each site to adjacent neighbors. For an open chain, unpaired bonds are left at each end of the chain, corresponding to effective localized $S=\frac{1}{2}$ objects. The model is believed to be a good representation of the ground state of the Heisenberg spin chain, except that the valence bonds are not restricted to form only between nearest-neighbor sites in the "real" wave function.

The existence of the edge states can also be understood from Berry phase consideration in the corresponding continuum nonlinear-sigma-model $(\mathrm{NL} \sigma \mathrm{M}){ }^{9}$ For a finite spin chain with length $L$, the Berry phase contribution to the effective action is 9,10

$$
S_{\mathrm{BP}}=\hbar \frac{S}{2} \int_{0}^{L} d \mathbf{x} \frac{\partial \Omega(\mathbf{x})}{\partial \mathbf{x}}
$$

where $\Omega(\mathbf{x})$ is the solid angle subtended by the close path on the surface of a unit sphere defined by the time evolution of a spin at $x$. Evaluating the integral we find that besides the usual (bulk) topological term $i S \int d \times d \tau F_{\mathbf{x} \tau},^{7-9}$ there is an extra contribution coming from the two limits of the integral, $S_{\mathrm{EP}}=\hbar(S / 2)[\Omega(L)-\Omega(0)]$, which can be interpreted as the Berry phases of two free spins with spin magnitude
$S / 2$, located at the end points $\mathbf{x}=0$ and $L$. The extra Berry phases modify the rule of angular momentum quantization at the end points, leading to effective $S=\frac{1}{2}$ ends states for $S=1$ chains.

The SBMFT can be interpreted as a variational approach with a VBS-type trial wave function, except that (1) valence bonds joining arbitrary sites on opposite sublattices are allowed, ${ }^{3,6}$ and (2) the constraint that there are $2 S$ spin- $\frac{1}{2}$ objects on each site is relaxed and is satisfied only on average. ${ }^{3-5}$ The theory can also be viewed as a generalization of the NL $\sigma \mathrm{M}$ where shortdistance fluctuations are included more properly. ${ }^{7,8}$ In this approach, the free energy of the spin system is a functional of the link variables $\Delta_{i j}=\left\langle\hat{Z}_{i \uparrow} \hat{Z}_{j \downarrow}-\hat{Z}_{i \downarrow} \hat{Z}_{j \uparrow}\right\rangle$, and the constraint fields $\lambda_{i}^{(A)}$ and $\lambda_{j}^{(B)} . \hat{Z}_{i \sigma}$ is a Schwinger-boson operator for spin $\sigma$ on site $i, j=i+\mu(\mu$ is a unit vector on the spin lattice), and $A$ and $B$ are sublattice indices. The ground state of the system is determined by minimization of the free energy with respect to the $\Delta$ and $\lambda$ variables $^{3,4}$ (the minimization of the free energy in $\lambda$ variables enforces the constraint of $2 S$ Schwinger bosons per site on average $\left.{ }^{3,4}\right)$. For general spin configuration like finite $1 \mathrm{D}$ spin chains minimization of free energy can be carried out only numerically. ${ }^{3}$ To derive an analytic theory, we assume that the variations of the order parameters $\Delta(\mathbf{x})$ and $\lambda(\mathbf{x})$ 's with respect to position $\mathbf{x}$ are slow (we shall make the meaning "slow" more clear in the following) and shall derive an approximate expression for the free energy in the lowest-order gradient expansion in these variables. In $1 \mathrm{D}$ and in the limit $S \rightarrow \infty$, such a calculation can be carried out analytically. The properties of the edge states will be studied in this continuum theory.

Following Read and Sachdev, ${ }^{7}$ we consider the representation where our systems have two sites per unit cell, and introduce the "uniform" and "staggered" field variables $\phi_{\mu}, Q_{\mu}, \theta$, and $A_{\mu}$, where

$$
\begin{gathered}
\Delta_{i, i+\mu}=\frac{1}{2}\left[\phi_{\mu}(i+\mu / 2)+Q_{\mu}(i+\mu / 2)\right] \\
\times e^{i\left[\theta_{\mu}(i+\mu / 2)+A_{\mu}(i+\mu / 2)\right]}, \\
\phi_{\mu}(\mathbf{x})\left[\theta_{\mu}(\mathbf{x})\right]=\phi_{-\mu}(\mathbf{x})\left[\theta_{-\mu}(\mathbf{x})\right], \\
A_{\mu}(\mathbf{x})\left[Q_{\mu}(\mathbf{x})\right]=-A_{-\mu}(\mathbf{x})\left[Q_{-\mu}(\mathbf{x})\right],
\end{gathered}
$$

$\mu= \pm \hat{X}$ and in momentum space,

$$
\begin{aligned}
& \theta_{\tau}(\mathbf{k})=\frac{1}{2}\left[\lambda^{(A)}(\mathbf{k})+\lambda^{(B)}(\mathbf{k})\right], \\
& A_{\tau}(\mathbf{k})=\frac{1}{2}\left[\lambda^{(A)}(\mathbf{k})-\lambda^{(B)}(\mathbf{k})\right] .
\end{aligned}
$$


The $\phi_{\mu}(\mathbf{x})$ field describes uniform antiferromagnetic correlations, whereas $Q_{\mu}(\mathrm{x})$ describes spin dimerization (spin-Peierls) effects. ${ }^{7} \theta_{\mu}(\mathbf{x})$ and $A_{\mu}(\mathbf{x})$ are fields describing the corresponding uniform and staggered phase fluctuations, respectively. It is also convenient to introduce the "relative" spin-dimerization field $q_{\mu}(\mathbf{x})=Q_{\mu}(\mathbf{x}) /$ $\phi_{\mu}(\mathbf{x})$. In terms of these variables, the free energy of the $1 \mathrm{D}$ spin chain in the large- $S$ limit and in lowest-order gradient expansion is (at $T=0) F=\int\left[F_{L}(\mathbf{x})+F_{G}(\mathbf{x})\right] d \mathbf{x}$, where

$$
\begin{aligned}
F_{L}(\mathbf{x})= & -\left\{(2 S+1)-(2 / \pi)\left[1-q_{\mu}(\mathbf{x})^{2}\right]^{1 / 2}\right\} \phi(\mathbf{x}) \\
& +\frac{1}{4} \phi(\mathbf{x})^{2}\left[1+q_{\mu}(\mathbf{x})^{2}\right]
\end{aligned}
$$

is the "local-density" part, and

$$
\begin{aligned}
F_{G}(\mathbf{x})= & \frac{\left[1-q_{\mu}(\mathbf{x})^{2}\right]^{1 / 2}}{6 \pi \phi(\mathbf{x}) m(\mathbf{x})^{2}} F_{\mathbf{x} \tau}^{2} \\
& +i\left[2 S+1-\gamma\left(q_{\mu}(\mathbf{x})\right)\right] q_{\mu}(\mathbf{x}) F_{\mathbf{x} \tau} \\
& +\frac{\phi(\mathbf{x})\left[1-q_{\mu}(\mathbf{x})^{2}\right]^{1 / 2}}{3 \pi m(\mathbf{x})^{2}}[\nabla \theta(\mathbf{x})]^{2} \\
& +(\text { higher-order terms }),
\end{aligned}
$$

is the part associated with the gradient expansion, $\gamma\left(q^{2}\right)=\left[m^{2}+\pi^{2}\left(1-q^{2}\right) / 4\right]^{-1 / 2}$. Notice that we have ignored the $\mu$ index for $\phi$ and $\theta$ fields for brevity. Notice also that we have already integrated out the fictitious $\theta_{\tau}$ field in Eq. (2), i.e., the average constraint per unit cell is already satisfied in the above expression. The staggered $A_{\tau}$ field is left behind for reasons which will become clear later.

The local-density part $F_{L}(\mathbf{x})$ is derived by evaluating the mean-field free energy directly with constant $\phi$ and $Q$. We have neglected terms of order $m(\mathbf{x}) \sim \exp (-\{1$ $\left.\left.-\left[q_{\mu}(\mathbf{x})\right]^{2}\right\}^{1 / 2} S\right)$ in (2a) where $m(\mathbf{x}) \phi(\mathbf{x})$ is the (local) Haldane gap. The gradient terms are derived by looking at Gaussian fluctuations around the mean-field state with given $\phi$ and $Q$. The calculation is a direct extension of the $1 / N$ expansion of Read and Sachdev ${ }^{7,8}$ (RS) around the SBMFT where they have considered fluctuations in the staggered channel (i.e., $Q$ and $A$ fields) around the mean-field state with $Q=0$ whereas we have considered mean-field states with nonzero $Q$ and also looked at fluctuations in both uniform and staggered channels. Our calculation is valid as long as $\mathbf{k}^{2}<m^{2}$ and $1-q_{\mu}^{2} \gg 1 / S$. The first term in $(2 b)$ is a direct generalization of the gauge field term $F_{\mathbf{x} \tau}^{2}$ in $\mathrm{RS}$ to the case with nonzero $Q$ $\left(F_{\mathbf{x} \tau}=\partial_{\mathbf{x}} A_{\tau}-\partial_{\tau} A_{\mathrm{x}}\right)$ and the second term represents the coupling between the gauge field and the spin-Peierls term $Q_{\mu}$ as in RS. Notice that $\gamma\left(q^{2}\right)<<S$ in the limit we are considering. The third term in (2b) involves the uniform phase $\nabla \theta$ and is absent in the RS treatment. In the gauge field language, $\nabla \theta$ can be considered as a $\mathbf{U}(1)$ gauge field in the uniform channel coupling to bosons on the two sublattices with same gauge charge. The existence of the nonzero $\Delta_{i j}$ order parameter implies that the (uniform) gauge symmetry is broken in the SBMFT, resulting in a Meissner effect on gauge field $\nabla \theta$ represented by the third term in (2b). The $\left(1-q^{2}\right)$ factors in (2b) can be understood by noting that in the limit $q_{\mu}=1$, the system is completely dimerized and phase fluctuations in different unit cells must be uncorrelated with each other. Notice that we have kept only $\nabla^{2}$ terms which are proportional to $(1 / m)^{2}$ in $(2 \mathrm{~b})$. The neglected gradient terms [e.g., $\left.(\nabla \phi)^{2}\right]$ have coefficients of order $S$ (or 1) and are small compared with the $(1 / m)^{2}$ terms in the limit we are considering.

The fluctuations represented by (2b) are coupled to the Schwinger bosons via the mean-field equations. ${ }^{3}$ For low-energy fluctuations, the most important effect is the coupling of the bosons to the (staggered) gauge fields, ${ }^{7,8}$ which can be represented most conveniently by introducing the continuum fields $\psi_{\sigma}=\left(Z_{\sigma}^{(A)}+Z_{\sigma}^{(B)^{*}}\right) / 2$ and $\pi_{\sigma}=\left(Z_{\sigma}^{(A)}-Z_{\sigma}^{(B)^{*}}\right) / 2$. Integrating out the $\pi_{\sigma}$ field we obtain 7,8

$$
\begin{gathered}
L_{\mathrm{eff}}=\frac{1}{2 \phi(\mathbf{x})}\left\{c(\mathbf{x})^{2}\left|\left(\partial_{\mathbf{x}}-i A_{\mathrm{x}}\right) \psi_{\sigma}\right|^{2}+\left|\left(\partial_{\tau}-i A_{\tau}\right) \psi_{\sigma}\right|^{2}\right. \\
\left.+\phi(\mathbf{x})^{2} m(\mathbf{x})^{2}\left|\psi_{\sigma}\right|^{2}\right\}
\end{gathered}
$$

where $c(\mathbf{x})=\left[\phi(\mathbf{x})^{2}-Q_{\mu}(\mathbf{x})^{2}\right]^{1 / 2}$ is the local spin-wave velocity. In $1 \mathrm{D} \mathrm{U}(1)$ gauge theory has linear Coulomb force and the coupling of the Schwinger bosons to the staggered gauge field results in confinement of spinons from opposite sublattices in pairs, leading to $S=1$ elementary excitations in infinite spin chains, instead of $S=\frac{1}{2}$ as in the naive mean-field theory. A finite spin chain can be represented in our formalism as a chain with broken bond $\Delta_{i, i+\mathrm{x}}=0$ at some sites $i$, where $\mathbf{x}$ is a unit vector. Using Eq. (1), we see that a broken bond can be represented by setting $\phi(i+\mathbf{x} / 2)=-Q_{\mu}(i+\mathbf{x} / 2)$, or $\phi\left(x_{0}\right)^{2}=Q_{\mu}\left(x_{0}\right)^{2}$ at some position $x_{0}$ in the continuum theory. The properties of a broken spin chain can be obtained by solving the corresponding continuum equation.

The exact solution of the continuum theory is difficult to obtain, but the general character can be understood quite easily. First we want to argue that the correct solution has $\phi\left(x_{0}\right) \neq 0$, so that the boundary condition can be expressed as $q_{\mu}\left(x_{0}\right)= \pm 1$. Physically the reason why a solution with finite $\phi\left(x_{0}\right)$ is preferred is that if $\phi\left(x_{0}\right)=Q_{\mu}\left(x_{0}\right)=0$, then from Eq. (1a) we see that we are actually breaking two adjacent bonds instead of one, which is not what we want. It is also not difficult to see by minimizing the free energy with respect to $\theta(\mathbf{x})$ that $\theta(\mathbf{x})=$ const. Furthermore, minimizing the energy with respect to $A_{\tau}$ we find that a staggered electric field $E=i F_{\mathbf{x} \tau}$ of magnitude $[2 S+1-\gamma(q)] / 2$ electric flux quanta will be induced by a nonzero $q_{\mu}= \pm 1$ at $x_{0}$. For infinite half-integral spin chains, Read and Sachdev ${ }^{7,8}$ have shown that the presence of a topological term in the effective action introduces electric field in the chains, and the coupling of electric field to $q_{\mu}$ leads to dimerization. In the present case, the situation is similar except that since the spin chain is finite, we do not have to look for a translational invariant (by two lattice sites) solution where the electric field is constant inside the chain. Instead, using the fact that the staggered $U(1)$ gauge field is coupled to the Schwinger bosons through an effective scalar electrodynamics action [Eq. (3)], one may construct a solution where the electric field is screened exponentially 
by exciting $[2 S+1-\gamma(1)] / 2$ bosons of appropriate charge at the end of the spin chain, in which the energy cost is only of order $[2 S+1-\gamma(1)] / 2 m \phi_{0}\left(\phi_{0}=\right.$ value of $\phi$ for infinite chain). Recall that $U(1)$ gauge theory is confining in 1D. Thus the screening charges are confined by the electric field at the end of the spin chain, forming the localized end states in the SBMFT. Notice that the "charge" of the localized spinon is distributed within a distance $\sim \xi(=$ correlation length) away from the end of the spin chain, ${ }^{3}$ implying that the electric field is also screened with a screening length $\sim \xi$. This and the result that $q_{\mu}(\mathbf{x}) \sim E(\mathbf{x})$ in the continuum theory imply that a residue exponentially decaying spin-Peierls effect will be found at the end of the spin chain together with the formation of the localized spinon state. This theoretical prediction is confirmed by our numerical solution of the broken spin chain in SBMFT. ${ }^{3,11}$ It was also confirmed more recently in exact diagonalization studies of short anisotropic spin chains where the dimerization effect is observed in the Haldane phase but not the large-D phase where end states do not exist. ${ }^{12}$ This physical picture also explains how the confined spinon pairs in the infinite spin chain are deconfined forming $S=\frac{1}{2}$ localized states at the ends of the finite spin chain.

It is interesting to point out that the end states in the VBS (Ref. 6) model can also be explained in a similar way. Suppose we want to "move" the free spin in the VBS model away from the end of the spin chain into the interior without exciting additional spinons. What would happen to the spin chain itself? It is easy to see that the only way this can be done in the VBS model is to have the region between the new free spin position and the end point completely dimerized. The energy cost of such a process will be proportional to the distance the free spin traveled, i.e., the spin will be confined linearly to the end point via a dimerization effect, which is exactly the physical picture obtained in SBMFT.

The continuum theory also reveals a major weakness of the SBMFT. Notice that $S$ is just a parameter in the SBMFT (Ref. 4) and that the electric flux induced at the end of the spin chain $(\sim[2 S+1-\gamma(1)] / 2)$ is not a quantized number, meaning that the number of spinons localized at the end of the spin chain is also not quantized. This defect is a direct consequence of the fact that the ground state in SBMFT is formed by condensation of pairs of spinons, $\left\langle\hat{Z}_{i \sigma} \hat{Z}_{j-\sigma}-\hat{Z}_{i-\sigma} \hat{Z}_{j \sigma}\right\rangle \neq 0$ and the constraint of $2 S$ bosons per site is only satisfied on average. The total number of spins in this mean-field ground state is not a good quantum number, which is reflected in the nonquantized nature of end states. In the limit $S \rightarrow \infty$, the number of electric flux quantz goes to $S$, suggesting that the correct number of spinons bound at the end of the chain should be equal to $S$ in the correctly quantized theory. Since each spinon carries spin $\frac{1}{2}$, the $S$ localized spinons together represent a free spin of magnitude $S / 2$, in agreement with prediction from the VBS model for higher spins.

Now we shall generalize our analysis to the 2D Heisenberg model on a square lattice. First let us point out that in $1 \mathrm{D}$, there exists always a gap in the excitation spectrum $^{4}$ (Haldane gap) and the identity of a localized spinon state depends on the existence of such a gap. In 2D, a gap exists only if the spin magnitude $S$ is less than a critical value $S_{c} \sim 0.19,{ }^{4,5}$ which is smaller than the smallest physical spin value $S=\frac{1}{2}$, meaning that physical Heisenberg antiferromagnets on a square lattice always have ordered ground states. In our following analysis, we shall consider a fictitious spin-disordered state with a gap in the spinon excitation spectrum. Nevertheless, this model is of interest because of its plausible relevance to the understanding of the high- $T_{c}$ superconductors ${ }^{5,7}$ and because the model may provide insights into the properties of other spin models which have a true spindisordered ground state. We shall look at edge excitations in this model.

The existence of nontrivial edge states in $2 \mathrm{D}$ can be visualized most easily by considering a 2D VBS model for $S=2$ spins where each site has four valence bonds joining to the four nearest-neighbor sites in the bulk state. For sites along the edge one valence bond is being removed leaving an effective $S=\frac{1}{2}$ Heisenberg spin chain along the edge of the system. As is well known a half-integer antiferromagnetic spin chain may be gapless or dimerized. ${ }^{13}$ Thus nontrivial edge states are expected to be formed in the 2D model. Notice that by the same construction, a 2D VBS model with $S=4$ has edge excitations corresponding to the $S=1$ spin chain. Thus in general the nature of the edge states should depend on the value of spin $S$. We shall see in the following that a naive SBMFT only predicts edge states with uniformly enhanced order parameter $\Delta_{i, i+\mu}$ along the edge independent of the value of $S$. This is, however, not surprising since a similar defect is also found in SBMFT for 1D spin chains. $S$ dependence of the edge states are obtained only after Berry phase effects are taken into account ${ }^{7,8}$ as we shall see later.

In 2D SBMFT, the spin system is characterized by the same bond and constraint variables $\Delta_{i, i+\mu}$ and $\lambda_{i}$ given by Eq. (1), except that $\mu$ can now take both $x$ and $y$ directions, i.e., we have totally eight continuum variables $\phi_{\mu}(\mathbf{x}), \theta_{\mu}(\mathbf{x}), Q_{\mu}(\mathbf{x})$, and $A_{\mu}(\mathbf{x})(\mu= \pm \hat{x}, \pm \hat{y})$ in the 2D theory. The free energy in the continuum limit can be derived in the same way as in 1D, and can be written as a sum of the local-density part $F_{L}(\mathbf{x})$ and "gradient" part $F_{G}(\mathbf{x})$. Unlike $1 \mathrm{D}, F_{L}(\mathbf{x})$ cannot be derived analytically. Instead we shall introduce the "sum" and "difference" fields $\phi_{s}(\mathbf{x})=\phi_{x}(\mathbf{x})+\phi_{y}(\mathbf{x})$ and $\chi_{a}(\mathbf{x})=\left[\phi_{x}(\mathbf{x})-\phi_{y}(\mathbf{x})\right] /$ $\phi_{s}(\mathbf{x})$ and shall assume that $q_{\mu}, \chi_{a}<1$, where $q_{\mu}=Q_{\mu} / \phi_{s}$. Expanding $F_{L}(\mathbf{x})$ in power series of $q$ and $\chi$ to second order we obtain the result

$F_{L}(\mathbf{x}) \sim-\phi_{s}(\mathbf{x})\left(2(2 S+1)-\left\{I_{0}-a_{1}\left[q_{x}(\mathbf{x})^{2}+q_{y}(\mathbf{x})^{2}\right]-a_{2} \chi_{a}(\mathbf{x})^{2}\right\}\right)+\left[\phi_{s}(\mathbf{x})^{2} / 4\right]\left\{1+\chi_{a}(\mathbf{x})^{2}+q_{x}(\mathbf{x})^{2}+q_{y}(\mathbf{x})^{2}\right\}$, 
where $I_{0}, a_{1}$, and $a_{2}$ are constants of order 1 .

The gradient part can be derived analytically to terms of order $1 / \mathrm{m}$ following a similar procedure as in 1D. To second order we obtain ${ }^{7,8}$

$$
\begin{aligned}
F_{G}(\mathbf{x})= & {\left[1 / 32 \pi m(\mathbf{x}) \phi_{s}(\mathbf{x})\right] F_{\mu \nu}^{2}+i N^{\prime}(q) q_{\mu}(\mathbf{x}) F_{\mu \tau} } \\
& +\left[\phi_{s}(\mathbf{x}) / 12 \pi m(\mathbf{x})\right]\left[\nabla_{\mu} \theta_{s}(\mathbf{x})\right]^{2},
\end{aligned}
$$

where $\theta_{s}(\mathbf{x})=1 / 2\left[\theta_{x}(\mathbf{x})+\theta_{y}(\mathbf{x})\right], \quad m(\mathbf{x}) \phi_{s}(\mathbf{x}) \sim[2.76$ $-2(2 S+1)] \phi_{s}(\mathbf{x})$ is the local spin gap, and $N^{\prime}(q)$ is a number of order 1 . We have assumed that $0<S_{c}-S \ll 1$ in the above derivation.

As in 1D, a 2D system with a boundary along the $y$ direction can be generated by setting $\Delta_{i, i+\hat{x}}=0$, at sites $i=\left(i_{0}, i_{y}\right)$, where $i_{0}$ is some given constant $x$ coordinate position, and $i_{y}$ runs from minus infinity to infinity. The corresponding boundary condition in the continuum theory is

$$
\begin{aligned}
& q_{x}(\mathbf{x})=1, \quad \mathbf{x}=\left(i_{0}-\frac{1}{2}, 2 n\right), \\
& q_{x}(\mathbf{x})=-1, \quad \mathbf{x}=\left(i_{0}+\frac{1}{2}, 2 n+1\right),
\end{aligned}
$$

where $n=$ integer and X's are position vectors of one sublattice. The above form is a consequence of the fact that the unit cell is "doubled" in the continuum theory [see Fig. (1)].

Solving the continuum equations as before (but now only to accuracy of order $q_{\mu}^{2}$ ), we find a nonzero electric field $E_{x} \sim N^{\prime}(1) \cos \pi y e^{-\pi x}$ and $E_{y} \sim N^{\prime}(1) \sin \pi y e^{-\pi x}$ along the boundary. The existence of alternating $E_{y}$ along the edge is the result of alternating $q_{x}(\mathbf{x})$ along the boundary. The coupling of $E_{y}$ to $q_{y}$ gives $q_{y} \sim E_{y}$. Notice that $E_{y}$ alternates in sign from one site to another, implying that $\Delta$ is enhanced uniformly along the edge but not dimerized. This behavior is also confirmed by numerical solutions of SBMFT in finite 2D systems. ${ }^{11}$

The effect of the Berry phase on solutions of the SBMFT has been discussed by Read and Sachdev ${ }^{7,8}$ for infinite $1 \mathrm{D}$ and 2D systems. ${ }^{10,14}$ For finite $1 \mathrm{D}$ systems extra Berry phases are left at the ends of the spin chains as discussed in our introduction, giving rise to the nontrivial end states. For 2D systems on the square lattice, these extra Berry phases along edges add up like antiferromagnetic spin chains with effective spin magnitude $S / 2$ along the boundaries.

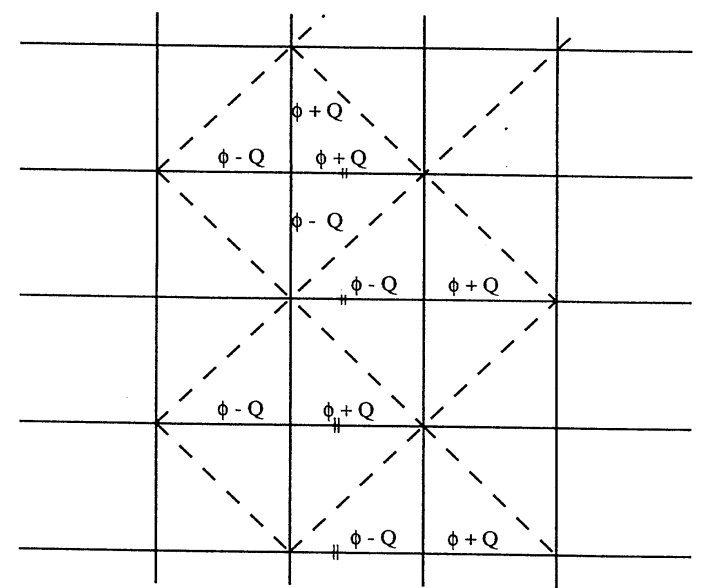

FIG. 1. Schematic representation of a $2 \mathrm{D}$ boundary. Solid lines represent the underlying square lattice, dashed lines represent the unit cells in SBMFT, and the broken bonds are labeled by the double dash. It is easy to see that $q_{x}$ alternates in sign along the boundary.

Following Read and Sachdev, ${ }^{7,8}$ a 1D spin chain (spin magnitude $S$ ) with Berry phase action can be viewed as a metastable state of the spin chain with a mean static electric field $E=S$ [mod(1)] flux quanta, leading to dimerization of half-integer spin chains. ${ }^{7,8}$ In the present case, the effective spin value of the boundary spin chain is $S / 2$, thus the boundary is dimerized for any spin $S \neq 2 n$ ( $n=$ integer) with degree of dimerization proportional to $S[\bmod (2)]$. Notice that this result is different from that suggested by the VBS model, which predicts that the boundary is dimerized except for $S=4 n$. The dimerization prediction for $2 \mathrm{D}$ boundaries is a mean-field result which is probably unreliable. However, the predicted periodic structure for the edge states is probably correct, since it follows just from the Berry phase computation. Notice that a similar analysis can also be carried out for other bipartite lattices (e.g., honeycomb lattice) and the resulting edge-state structures will generally be different. Details of these calculations will be given in a separate paper.

The author thanks A. Tanaka for pointing out the important difference in end states between the Haldane phase and large-D phase.
${ }^{1}$ M. Hagiwara, K. Katsumata, I. Affleck, B. I. Halperin, and J. P. Renard, Phys. Rev. Lett. 65, 3181 (1990).

${ }^{2}$ S. H. Glarum, S. Geschwind, K. M. Lee, M. L. Kaplan, and J. Michael, Phys. Rev. Lett. 67, 1614 (1991).

${ }^{3}$ T. K. Ng. Phys. Rev. B 45, 8181 (1992).

${ }^{4}$ D. P. Arovas and A. Auerbach, Phys. Rev. B 38, 316 (1988).

${ }^{5}$ A. Auerbach and D. P. Arovas, Phys. Rev. Lett. 61, 617 (1988).

6I. Affleck, T. Kennedy, E. H. Lieb, and H. Tasaki, Phys. Rev. Lett. 59, 799 (1987).

${ }^{7}$ N. Read and S. Sachdev, Phys. Rev. Lett. 62, 1694 (1989).

${ }^{8}$ N. Read and S. Sachdev, Phys. Rev. B 42, 4568 (1990).
${ }^{9}$ F. D. M. Haldane, Phys. Lett. 93A, 464 (1983); I. Affleck, Nucl. Phys. B257, 397 (1985).

${ }^{10}$ X. G. Wen and A. Zee, Phys. Rev. Lett. 61, 1025 (1988); E. Fradkin and M. Stone, Phys. Rev. B 38, 7215 (1988); T. Dombre and N. Read, ibid. 38, 7181 (1988).

${ }^{11}$ T. K. Ng (unpublished).

${ }^{12}$ A. Tanaka, M. Oshikawa, and Y. Hatsugai (unpublished).

${ }^{13}$ See, for example, I. Affleck, J. Phys. Condens. Matter 1, 3047 (1989).

${ }^{14}$ F. D. M. Haldane, Phys. Rev. Lett. 61, 1029 (1988). 